\title{
Der Straftatbestand des Ausnutzens einer schutzlosen Lage des Opfers ist nicht zu unbestimmt.
}

\author{
Das BVerfG zu §§ 177 Abs. 1 Nr. 3 StGB, Art. 103 GG, Bestimmtheitsgrundsatz \\ und Grundsätze eines liberalen und rechtsstaatlichen Strafrechts - BVerfG, \\ Beschluss v.1.7.2004 - 2 BvR 568/04 = NStZ 2005, 30 = NJW 2004, 3768 \\ zu BGH 2 StR 351/03 = NStZ 2004, 440.
}

Monika Frommel

\section{Sachverhalt:}

D ie zum Tatzeitpunkt 9-Jährige Geschädigte begleitete den Angekl., ihren als LKWFahrer tätigen Groß-vater, im Jahre 2002 in den Ferien bei Auslieferungsfahrten. Bei der ersten Tat begaben der Angekl. und die Geschädigte sich nach dem Besuch eines Autobahnrestaurants gegen 21:00 Uhr in den auf einem Autobahnparkplatz abgestellten LKW des Angekl., um dort in zwei übereinanderliegenden Schlafkojen zu übernachten. Nachdem die sich mit einem T-Shirt und Slip bekleidete Geschädigte eingeschlafen war, fasste der Angekl. den Entschluss die Abgeschiedenheit der Fahrerkabine zu nutzen und griff dem Kind unter das T-Shirt, streichelte ihre Brust und drückte diese zusammen. Das deswegen erwachende Mädchen entzog sich der Situation indem sie sich wegdrehte, woraufhin der Angekl. von ihr abließ.

Bei der zweiten Tat, eine Woche später, näherte sich der Angeklagte erneut unter Ausnutzung der abgeschiedenen Lage dem Mädchen, das gegen 22:00 Uhr in der Schlafkoje im Begriff war einzuschlafen. Wiederum drückte der Geschädigte die Brust des Mädchen zusammen und griff ihr sodann unter ihre Leggin um zunächst an der Scheide des Mädchens zu reiben und sodann zwei Finger bis zum Mittelgelenk in die Scheide einzuführen. Die sich im Halbschlaf befindliche Geschädigte erwachte und zog die Hand des Angekl. aus ihrer Hose, der daraufhin von der Geschädigten abließ.

Mit der Verfassungsbeschwerde angegriffen wurde nicht die Verurteilung wegen sexuellen Missbrauchs eines Kindes (unstrittig gegeben), sondern nur die zusätzliche Verurteilung wegen sexueller Nötigung beim ersten Übergriff sowie die Annahme einer sexuellen Nötigung/Vergewaltigung beim zweiten Übergriff auf das Kind.
Vorgetragen wurde vom Beschwerdeführer, die Auslegung des neu eingefügten Merkmals verstoße gegen den Bestimmtheitsgrundsatz, da eine lediglich gegen den Willen des Opfers durchgeführte sexuelle Handlung keine Nötigung sei. Gestützt auf die insb. von Fischer im Standard-Kommentar Tröndle/Fischer vertretene, aber vom 2 . Strafsenat, dem er vorsitzt, nicht übernommene Doktrin, wonach eine Nötigung begriffsnotwendig ein zweiaktiges Vorgehen voraussetze. Dieser angeblich unstreitige strafrechtsdogmatische Begriff der Nötigung sei aber vom Gesetzgeber bei der Neufassung des § 177 StGB im Jahre 1997 verwendet worden. Die Auslegung durch die höchstrichterliche Rechtsprechung verstoße daher gegen den Wortlaut und damit gegen den Bestimmtheitsgrundsatz. Dieser lautet: "Nötigen unter Ausnutzen einer schutzlosen Lage«.

\section{Zur Vorgeschichte der Erweiterung des}

Tatbestandes in § 177 Abs. 1 Nr. 3 StGB im Jahre 1997

Der neue Einheitstatbestand der sexuellen Nötigung / Vergewaltigung trat nach mehr als zehn Jahren und ziemlich ermüdenden Debatten im Juli 1997 in Kraft, welche heute in dieser Form undenkbar wären. Stein des Anstoßes war über Jahre der von der Rechtsprechung deliktspezifisch unterschiedlich ausgelegte Gewaltbegriff. Er wurde bei Sitzblockaden weit (§ 240 StGB) und bei sexueller Nötigung und Vergewaltigung sehr restriktiv gehandhabt. Auch konkludente Drohungen mit Gewalt wurden nicht angemessen berücksichtig, sondern als nur psychische Beeinträchtigung abgetan ${ }^{1}$. Durch die Erweiterung des Tatbestandes der sexuellen Nötigung schuf die Gesetzgebung nicht zuletzt deswegen eine zusätzliche Tatmodalität, welche wie ein Auffangtatbestand wirken sollte. Der BGH setzte unmittelbar nach der Reform dieses Ziel um und legte die Ausnutzungsvariante in BGH St 45, 256 weit aus. Diese Rechtsprechung wird mittlerweile trotz der kritischen Kommentierung in fast allen Kommentaren von allen Strafsenaten (mit Einschränkungen beim 4. Strafsenat) vertreten, vgl. BGH, NStZ 2004, 440. Aber der weltanschaulich geführte Kampf wurde dennoch - bis in die jüngste Vergangenheit - fortgesetzt.

Ohne das empirische Material zur Kenntnis zu nehmen, das die Gesetzgebung 1997 zur Tatbestandserweiterung motiviert hatte, nämlich die spezifischen Rahmenbedingungen von Ohnmacht und Dominanz in gestörten Geschlechterverhältnissen, begann umgehend nach der Einfügung der Ausnutzungsvariante in $\S 177$ Abs. 1 Nr. 3 ein m.E. nur vordergründig mit strafrechtsdogmatischen Argumenten ausgetragener Streit darüber, ob der erheblich weiter gefasste Tatbestand angemessen sei. Im Gegensatz zur Nötigung oder dem Raub enthält der reformierte Tatbestand der sexuellen Nötigung nämlich nicht nur die Nötigungsmittel »Gewalt« und "Drohung «, sondern eine weitere Variante, das Ausnutzen einer schutzlosen Lage des Opfers durch den Täter. Der Grund für diese Erweiterung waren die in zahlreichen Strafverfahren der 1970er bis 1990er Jahre zutage getretenen Unstimmigkeiten, die damit zusammen hingen, dass eingeschüchterte Opfer sexueller Übergriffe sich nicht wehrten und ihre Passivität umschreiben als »starr vor Schreck«. Die Beschuldigten (und ihre anwaltlichen Vertreter) deuteten dies als »Einwilligung « um, Gutachter bescheinigten damals sogar eine Art geschlechtsspezifische Situationsverkennung, und die Revisionsgerichte in den 1970-1990er Jahren tendierten dazu, derartige Konstellationen als "nur « psychische Zwangslagen einzustufen, die weder unter den Gewaltbegriff noch den der Drohung mit einer gegenwärtigen Gefahr für Leib oder Leben in $\S \S 177,178$ StGB a.F. zu subsumieren seien. 
Nach empirischen Studien ${ }^{2}$ handelte es sich dabei immerhin um etwa die Hälfte aller Ermittlungsverfahren und um etwa $20 \%$ der durchgeführten Strafverfahren. Die Gesetzgebung änderte daher die Anforderungen an die sexuelle Nötigung und schuf eine flexibel zu handhabende Strafzumessungsnorm der Vergewaltigung (der sog. Einheitstatbestand des $\S 177$ Abs. 1 und 2, welcher die $\$ \S 177,178$ a.F. ersetzte). Die Rechtsprechung implementierte das Reformgesetz, legte die Ausnutzungsvariante also weit aus und provozierte in der strafrechtsdogmatischen Literatur begriffsjuristische Bedenken. Aus strafrechtsdogmatischer Sicht könne sich eine "Nötigung « begrifflich nicht in einer bloßen Willensbeugung erschöpfen, sondern müsse zweiaktig erfolgen, d.h. zunächst müsse der Täter mit Gewalt oder Drohung auf das Opfer einwirken und dann den Nötigungserfolg herbeiführen. Insbesondere Fischer, der Kommentator des am häufigsten benutzten Kurzkommentars (Tröndle/ Fischer), machte sich stark für eine dieser Doktrin folgenden Korrektur des Gesetzes durch Auslegung, fand aber innerhalb den BGH Richterinnen und Richter keine Mehrheiten. Gegen ihn wurde (in seinem eigenen Senat) insbesondere ausgeführt, dass die Gesetzgebung schließlich genau diese Doktrin für den Spezialfall der sexuellen Nötigung habe ändern wollen (historische Auslegung). Denn schließlich hätten vor der Reform 1997 eine große zahl von Kritiker moniert, dass die Rechtsprechung bei den sexuellen Gewaltdelikten - gegen ihre Tendenz bei der einfachen Nötigung und auch beim Raub einen deliktspezifisch zu eng ausgelegten Gewaltbegriff benutze. Diesen abzuschaffen sei u.a. der Sinn der Reform gewesen, so dass sich nun eine Korrektur des gesetzgeberischen Willens verbiete. Im übrigen gibt auch Fischer die Unangemessenheit der Rechtsprechung zu den begriffen der Gewalt und der Drohung in $\S 177$ Abs. 1 Nr. 1 und 2 StGB zu (seit der Übernahme der Kommentierung in der 49. Aufl. 1999). Aber er wollte die von der Rechtsprechung geschaffenen Lücken auf eine andere Weise schließen - durch allmählichen Rechtswandel.

Zur Erklärung des Vorwurfs, der Gewaltbegriff sei bei Sitzblockaden weit (bis zur Korrektur der sog. Läpple-Entscheidung BVerfGE 73, 206 durch BVerfGE 92, 1) und bei sexuellen Übergriffen eng ausgelegt worden, sei an die Kontroversen in den 1980er Jahren erinnert, die sich immer wieder an vom BGH bestätigten spektakulären Freisprüchen entzündet hatten, etwa dem sog. Wuppertaler Lehrherrn-Fall, einem erzwungenen Beischlaf in einer vom Angeklagten herbeigeführten Zwangslage, um ein noch sehr junges und von ihm abhängiges Mädchen gefügig zu machen. Angesichts der erkannten Aussichtslosigkeit von Gegenwehr, entschied sich die junge Frau, wenigstens auf die Empfängnisverhütung zu achten. Diese minimale Kooperation ließ nach Ansicht der Gerichte »Gewalt« entfallen. ${ }^{3}$
Aber wie so oft ist das erklärte Ziel der Reform 1997 klarer als die Begründung in den Gesetzesmaterialien. Dort sind insbesondere keine Ausführungen darüber enthalten, ob das systematische Problem gesehen wurde, das sich daraus ergibt, dass normalerweise der Nötigungserfolg (Beispiel: Wegnahme der Handtasche durch einen »Handtaschenräuber «) nicht mit der Nötigungshandlung (heftiges Ziehen an der Tasche mit körperlicher Kraft, d.h. »Gewalt «) zusammen fallen darf. Bei Sexualkontakten gegen den Willen einer sich nicht wehrenden Frau ist es aber schwierig, die Nötigungshandlung begrifflich von der erzwungenen sexuellen Handlung abzugrenzen, da zwar der erzwungene Beischlaf »körperliche Kraft« kosten mag, diese aber während, nicht davor entfaltet wird, wenn es gelingt, das Opfer zur Duldung zu bewegen. Folglich gibt es eine Vielzahl von Fallkonstellationen, bei denen die Doktrin der »begriffsnotwendigen Zweiaktigkeit der Nötigung in Schwierigkeiten geraten muss, man denke nur an überraschende, überrumpelnde und solche Handlungen gegen den Willen eines zur Gegenwehr nicht fähigen Opfers. Dem sexuellen Übergriff geht eben in etwa $50 \%$ aller Fälle keine vorherige Gewalt voraus und auch keine explizite Drohung (mit Leibund Lebensgefahr). Zwar hätten gutwillige Gerichte in solchen Konstellationen mit der Konstruktion einer konkludenten Drohung operieren können, aber sie haben dies nicht getan und außerdem hilft auch eine solche Argumentation nicht immer weiter, insbesondere dann nicht, wenn das Opfer ohnehin dem Zugriff des Täters schutzlos ausgeliefert ist, also gar nicht mehr »konkludent« bedroht werden muss ${ }^{4}$.

Die Beschwerdeführer der abgewiesenen Verfassungsbeschwerde wollen alle diese Konstellationen, in denen keine brachiale Gewalt ausgeübt und nicht explizit gedroht worden ist, das Opfer auch nicht aus Angst vor weiteren körperlichen Angriffen passiv bleibt, unter den Begriff des sexuellen Missbrauchs subsumieren, wissend dass immer dann, wenn das Opfer kein Kind mehr und auch nicht so behindert ist, dass es über § 179 StGB geschützt wird, neue Lücken auftreten werden. Durch den Beschluß des BVerfG ist dieser Streit nun vorläufig beendet, was meint, dass die wichtigste Teilreform der Sexualdelikte zu einer nicht mehr rechtlich angreifbaren höchstrichterlichen Rechtsprechung geführt hat.

\section{Anmerkung:}

Die Beschwerdeführer stützen sich auf eine Mindermeinung, welche sich bisweilen wie eine hM präsentiert, schon deshalb weil so gut wie alle namhaften Kommentare ihr folgen. ${ }^{5}$ Danach müsse auch nach der Reform die sexuelle Nötigung nach $\S 177$ Abs. 1 Nr. 3 StGB "Zweiaktig « konstruiert werden. ${ }^{6}$ Zunächst müsse eines der spezifischen Nötigungsmittel auf
Seiten des Täters eingesetzt werden, welches dann den Nötigungserfolg herbeiführe - hier das Dulden oder die Vornahme einer sexuellen Handlung auf Seiten des Opfers (Wortlautargument und systematisches Argument, begründet mit der begriffslogischen Struktur aller Nötigungsdelikte). Da das Gesetz den Begriff des »Nötigens « ausdrücklich im Zusammenhang mit den ersten beiden Varianten von § 177 Abs. 1 verwende, müsse dies auch für die dritte Variante gelten. Über die schutzlose Lage des Opfers hinaus, sei daher eine eigenständige Zwangshandlung seitens des Täters nötig ${ }^{7}$, anderenfalls handele es sich lediglich um die bloße Missachtung des entgegenstehenden Willens eines anderen, was aber noch keine Nötigung darstelle 8 . Darüber hinaus argumentiert diese Position noch mit Blick auf eine sachgerechte Abgrenzung zu den Missbrauchsdelikten (§§ 176 und 179 StGB) systematisch. § 176 StGB laufe leer, da beim Verzicht auf eine eigenständige Nötigungshandlung kaum Fälle des sexuellen Missbrauchs von Kindern denkbar seien, in denen nicht zugleich auch eine sexuelle Nötigung gegeben sei, da diese Taten typischerweise gegen den Willen der Opfer und in Abwesenheit anderer Personen, also in einer schutzlosen Lage, vollzogen werden ${ }^{9}$. Allerdings lässt diese zweite Argumentation zweierlei außer Acht: Zum einen ist das von $§ 176$ StGB geschützte Rechtsgut ein abstraktes Gefährdungsdelikt, es untersagt somit jeden sexuellen Kontakt mit Kindern, nicht nur »unerwünschten «, eine Willensbeugung des Kindes ist daher gerade nicht erforderlich. Zum anderen war schon vor der Einführung des § 177 Abs. 1 Nr.3 StGB allgemein anerkannt, dass bei erzwungenen Sexualkontakten mit Kindern sexuelle Nötigung und sexueller Missbrauch von Kindern tateinheitlich vorliegen ${ }^{10}$ und sich nicht etwa ausschließen.

Die herrschende Meinung in der Rechtsprechung (und zumindest die Literatur, welche spezialisiert zum reformierten Sexualstrafrecht gearbeitet hat, etwa neben den schon genannten Autoren das Lehrbuch von Laubenthal) geht demgegenüber davon aus, dass die Gesetzgebung bewusst keinen für alle Nötigungsdelikte geltenden weiten Begriff der Gewalt und Drohung normieren wollte (verworfen wurde etwa der Vorschlag, im Wege einer Legaldefinition den Gewaltbegriff zu erweitern). Man hat sich stattdessen darauf beschränkt hat, die spezifische Situation von Opfern sexueller Übergriffe zu verbessern, ansonsten aber die hohen Anforderungen an die finale Verknüpfung von Gewalt und Nötigungserfolg zu belassen. Die Berufung auf irgendwelche sachlogischen Strukturen eines deliktsunspezifischen Nötigungsbegriffes, der zwingend voraussetze, dass die Nötigungshandlung und der Nötigungserfolg auch bei § 177 StGB begrifflich sauber zu trennen sei, ist daher äußerst fragwürdig. Im übrigen kann es nun einmal sein, dass auch eine Gewalthandlung, wenn sie überraschend erfolgt 
und dem Täter sofort eine körperliche Berührung ermöglicht, welche als Nötigungserfolg im Sinne der sexuellen Nötigung (sexuelle Handlung) gelten muss, einaktig ist (überrumpelnde sexuelle Übergriffe mit physischer Kraftentfaltung). Dasselbe gilt für konkludente Drohungen gegenüber eingeschüchterten Opfern. Alles dies war der Gesetzgebung 1997 bekannt und sie hat - gestützt auf empirische Studien - bewusst einen weiten Begriff der sexuellen Nötigung formuliert und diesen als 3. Alternative in $\S 177$ Abs. 1 eingefügt. Dies bedeutet, dass jedenfalls sexuelle Nötigungen - je nach Vorgehensweise des Täters - zwei- oder einaktig sein können. ${ }^{11}$ Zur Verwirklichung des Ausnutzungstatbestandes genügt daher bereits die Vornahme einer sexuellen Handlung gegen den Willen des Opfers in einer körperlich wirkenden Zwangslage (unter Ausnutzung einer schutzlosen Lage). Einer darüber hinausgehenden gesonderten Nötigungshandlung bedarf es demnach nicht, diese erschöpft sich vielmehr in der Vornahme der sexuellen Handlung in einer Zwangslage gegen den Willen des Opfers. Verlangt wird also auch bei der Ausnutzungsvariante ein körperlich wirkender Zwang. Ausgeschlossen sind Fälle, in denen das Opfer - ohne um sein körperliches Wohl besorgt zu sein, lediglich zur Vermeidung von sozialen Nachteilen - einen an und für sich nicht erwünschten Sexualkontakt über sich ergehen lässt. Diese können aber unter den Vergehenstatbestand des $\S 240$ Abs. 4 Nr. 1 StGB (etwa bei einer Drohung mit einem empfindlichen Übel) fallen. ${ }^{12}$ Insgesamt ergibt dies ein abgestuftes System von Straftatbeständen, welche je nach Konstellation eine angemessene Intervention erlauben.

Das BVerfG stellt nun fest, dass die weite Auslegung der 3. Alternative nicht den möglichen Wortsinn des Wortes "Nötigen « überschreitet und der Tatbestand der sexuellen Nötigung dadurch weder konturlos noch unbestimmt wird. Dabei lässt es bewusst offen, ob die höchstrichterliche Rechtsprechung "richtig « ist. Sie verstoße aber jedenfalls nicht gegen das Bestimmtheitsgebot.

Nach der hier vertretenen Sicht macht es durchaus Sinn, in einer so klar definierbaren Täter-Opfer-Situation auch diffuse Zwangssituationen zu erfassen, wenn entweder körperlich wirkender Zwang oder eine Drohung (ggf. nur im Sinne des Vergehenstatbestandes $\S 240$ Abs. 4 Nr. $1 \mathrm{StGB})$ vorliegt. Nur dann kann das sexuelle Selbstbestimmungsrecht symbolisch gestärkt und eine "Verhandlungsmoral « auch gegen diejenigen, die noch in alten Kategorien denken, durchgesetzt werden. Sofern also der erweiterte Tatbestand der sexuellen Nötigung nicht ausufernd ausgelegt wird und sichergestellt ist, dass Entscheidungen in diffusen Zwangslagen, insb. in ökonomischer oder sozialer Abhängigkeit nicht einfach als »Willensbeugung « umgedeutet und unter die dritte Alterna- tive subsumiert werden, kann eine verstärkte Kontrolle von nicht brachial gewaltförmigen sexuellen Nötigungen mit strafrechtlichen Mitteln angemessen und effektiv sein.

Wie so oft ist die höchstrichterliche Rechtsprechung (noch) nicht ganz einheitlich. Der 4. Strafsenat des BGH höhere Anforderungen (zumindest an die tatrichterliche Begründung) als die übrigen Strafsenate ${ }^{13}$. Auch so gesehen ist dieser Beschluss möglicherweise für die künftige Umsetzung des weiten Tatbestandes der sexuellen Nötigung wichtig. Aber nicht verschwiegen werden soll auch an dieser Stelle, dass es mittlerweile Regelungsbereiche gibt, in denen Strafrecht nur noch als (Schein)Lösung für strukturelle Probleme eingesetzt wird. Aus diesem Grund dokumentieren wir in diesem Heft auch eine Auseinandersetzung mit dem Phänomen der sog. "Zwangsprostitution", ein moralisch aufgeladener Begriff, der in jüngster Zeit zunehmend dazu benutzt wird, um eine - aus welchen gründen auch immer - unerwünschte Arbeitsmigration (in nahezu willkürlich herausgegriffenen Fällen) zu kriminalisieren. Auch hier wird mit einem extrem weiten und konturlosen Begriff der »Ausnutzung einer Zwangslage « gearbeitet (so in $\S 232$ in der Fassung vom 19.02.2005, der gerade nicht erzwungenen, sondern nur organisierten Vermittlung in die Prostitution). Geleugnet wird hier nicht ein Regelungsbedarf, aber es stünden andere und effektivere rechtliche Instrumente zur Verfügung (EU-Arbeitsrecht) zum Schutz abhängig arbeitender MigrantInnen. Offenbar verführen weit gefasste Tatbestände in einem akzeptierten Regelungsbereich $\mathrm{zu}$ »mehr vom selben« in höchst fragwürdigen Kontexten. Aber dies ist ein anderes Kapitel ${ }^{14}$.

\section{Fußnoten}

1 Im jüngsten Heft der NStZ kann man nachlesen, wie hoch der 4. Strafsenat am 1.07.2004, d.h. zeitgleich mit der Entscheidung des BVerfG, die Anforderungen schrauben wollte, und eine Seite weiter ist eine BGH-Entscheidung zum alten Recht abgedruckt, BGH NStZ 2005, 268, welche alles bestätigt, was ich 1993 als » Rechtsverweigerung « charakterisiert habe: Frommel, Die höchstrichterliche Rechtsprechung zur Vergewaltigung und sexuellen Nötigung - unverfroren und unbeirrbar, in: Vom Guten, das noch stets das Böse schafft, Böllinger/Lautmann (Hrsg.), 1993; dies., Rechtsprechung statt Rechtsverweigerung, NK 1993, 22.

2 Udo Steinhilper zeigte 1986, dass etwa in der Hälfte aller Ermittlungsverfahren die damals gültigen $\S \S 177,178$ StGB a.F. sehr eng ausgelegt worden sind und deshalb schon bei der Anklageerheung Schwierigkeiten bereitet haben, so dass es im Vergleich zu anderen Delikten deutlich überhöht zu Einstellungen nach § 170 Abs. 2 StPO (fehlender Tatnachweis) kam, vgl. hierzu die ausführliche die Rezension von Frommel MSchrKrim 70/1987, 120 f

3 Ein ehemaliger Bundesrichter verteidigte die BGH-Entscheidung in der ZRP, Frommel replizierte: Mösl, ZRP 1989, 49, Replik in: ZRP 1989,
232; dies. »Rechtsprechung statt Rechtsverweigerung «, NK 3/1993, 22.

4 Aber sie gingen dann ein Risiko ein, von Revisionsrichtern aufgehoben zu werden. Selbst zur Ausnutzungsvariante wiederholt sich dieses Muster beim 4. Strafsenat in seiner Entscheidung vom 1.07. 2004 - BGH NStZ, 2005, 267. Auch Susanne Folkers unterschätzt die Probleme, die Instanzgerichte vor der Reform hatten und meint in ihrer auch sonst auffallend wenig sorgfältig gearbeiteten Anmerkung eine Ehrenrettung für die Rechtsprechung vergangener Zeiten liefern zu müssen, dies, NStZ 2005, $181 \mathrm{ff}, 183$ linke Sp. oben.

5 Mit Ausnahme der Kommentierung im Münchner Kommentar (Renzikowski) und im NomosKommentar (Frommel). Staatsanwältin Folkers hat dementsprechend auch in ihrer Besprechung (NStZ 2005, $181 \mathrm{ff}$.) den Eindruck, als habe der BGH NStZ 2004, 440 und das BVerfG eine alte Tradition (in ihren Augen »ohne Not«) beendet. In Wahrheit hat der BGH sofort nach der Reform in BGH St 45, 253 ff. die Ziele der Reform umgesetzt. Die herkömmlichen Kommentare versuchten zwar diese Tendenz zu unterlaufen, aber ohne Erfolg, vgl. hierzu im einzelnen Frommel, Nomos-Kommentar zum StGB 2001 und 2. Aufl. $2005 \mathrm{zu} \mathrm{§}$ 177, insb. Rdnr. 1, 6 ff., 20 ff., 48 ff (der 2. Aufl.).

6 Fischer, NStZ 2000, 142 (143) und seit der 49. Aufl. des Tröndle/Fischer, StGB, vgl. dort 52. Aufl. (2004) § 177, Rn. 46; ihm folgen Schönke/Schröder-Lenckner/Perron, StGB, 26. Aufl. (2001), § 177, Rn. 11 und Güntge, NJW 2004, S. 3750 ff., 3752. Folkers a.a.O. folgt den genannten Autoren, aber sie scheint die Entscheidung falsch $\mathrm{zu}$ verstehen. Gegenstand war nicht die Fallgruppe des Missbrauchs einer Schlafenden, da das Mädchen aufgewacht ist, die sexuelle Handlung also bewusst erlebt hat.

7 Tröndle/Fischer $\S 177$ Rn. 39 und 46.

8 So auch Graul, JR 2001, S. 117, 118.

9 Güntge a.a.O. S. 3751; ähnlich Tröndle/Fischer Rn. 37.

10 So schon BGH bei Dallinger, MDR 74, 546. Seit dem 1.04.2004 gilt dies auch für das Zusammentreffen von Missbrauch Widerstandsunfähiger und sexueller Nötigung.

11 So explizit BGH NStZ 2004, 440; NK-Frommel, 2001 \& 177, Rn. 21f und $45 \mathrm{ff} . ;$ in der demnächst erscheinenden 2. Aufl., 2005, wird der spezifische Begriff der sexuellen Nötigung präzisiert. Danach muss die Ausnutzungsvariante als eine Form des körperlich wirkenden Zwangs interpretiert werden, um sie von diffusen Zwangslagen zu unterschieden, bei denen das Opfer lediglich soziale Nachteile befürchtet.

12 Entschieden vom BGH NStZ 2003, 534 = NJW 2003, 2250. Das Opfer muss sich also aus Sorge um sein körperliches Wohl dem Ansinnen des Täters beugen. Ein struktureller Gewaltbegriff wird auch mit der Reform 1997 nicht konstruiert.

13 BGH NStZ 2005, 267 (zur Vergewaltigung einer Schutzbefohlenen im familiären Bereich).

14 Beiträge in diesem Heft zum Menschenhandel. 